\title{
I COMPOSTI TRINOMINALI DEL TIPO RELAZIONE GOVERNO-SINDACATI IN ITALIANO (DATI DA CORPUS E TEORIA A CONFRONTO) ${ }^{1}$
}

\author{
Jan RADIMSKÝ \\ Università della Boemia meridionale, České Budějovice
}

\begin{abstract}
En): Italian has two different types of compounds that may be described as trinominal: (a) the type exemplified as direzione ufficio acquisti and the type exemplified as relazione governo-sindacati. In both cases, the N2-N3 component modifies the head noun (N1), but the difference lies in the fact that in the former type, the relation between N2 and N3 is subordinate, while it is coordinate in the latter type. The aim of this paper is to collect representative data concerning the latter type of compounds and to analyze their internal structure. Our analysis is based on the hypothesis that the internal structure of these compounds is governed by the head noun N1: thus, corpus data were used to identify several key properties of the head noun that determine the internal structure and the interpretation of trinominal compounds.
\end{abstract}

Keywords (En): Word Compounding, Italian, Relational Compound, Coordinate Compound.

\section{Introduzione}

Solitamente (cf. A. Bisetto in: GROSSMANN-RAINER, 2004) si distinguono in italiano due tipi di composti che possono essere considerati trinominali $(\mathrm{N}-\mathrm{N}-\mathrm{N})$ : il tipo (a) direzione ufficio acquisti e il tipo (b) relazione governo-sindacati (BISETTO A., 2004 : 42, 39). In entrambi i tipi, il complesso N2-N3 sembra fungere da modificatore ${ }^{2}$ del $\mathrm{N} 1$; una differenza importante consiste però nella natura della relazione sintattica tra i costituenti N2 e N3, che è subordinativa nel tipo (a) e coordinativa nel tipo (b). Purtroppo, la descrizione citata è poco dettagliata in entrambi i casi e la ricerca bibliografica non ci ha consentito di reperire studi più approfonditi per l'italiano. Sembra perciò valida la constatazione di Laurie BAUER (2009:355), secondo cui "most discussions of $N+N$ compounding, for instance, will provide some examples of the construction, but few will give an exhaustive analysis of the types of semantic relationship that may hold between the two nouns." Il presente articolo si pone come obiettivo quello di raccogliere dati rappresentativi sui composti trinominali del tipo (b) nel corpus ItWac e di descrivere le relazioni sintattiche e semantiche tra i costituenti, mostrando che la tipologia di queste relazioni è indissolubilmente legata alle proprietà del nome testa (N1).

Vedremo che i composti trinominali del tipo (b), chiamati relazionali (relational compounds) da B. WÄLCHLI (2005 : 7), rappresentano in realtà una categoria relativamente variegata. In conformità con la classificazione proposta in linguistica generale da L. BAUER $(2008,2009)$ vi includeremo, accanto ai coparticipant compounds (fr. des traités franco-russes), anche i composti chiamati

\footnotetext{
${ }^{1}$ Tengo a ringraziare Antonietta Bisetto per i suoi preziosi commenti e suggerimenti. Il presente articolo è stato realizzato grazie al sostegno finanziario del progetto GAČR 13-00496S della Fondazione scientifica ceca.

${ }^{2}$ Useremo il termine modificatore nel senso più generale per far riferimento all'espansione di qualsiasi natura.

Echo des études romanes X/1-2, $2014 \quad 35$ -

ISSN: 1804-8358(Online)
} 
da Bauer translative compounds (fr. vol Paris-Rome). Secondo BAUER (2008 : 1112), la differenza principale tra i composti del tipo translative e co-participant consiste nel fatto che nei primi la relazione tra i componenti è semanticamente orientata con un punto di "partenza" e un punto di "arrivo", facendo sì che il loro ordine rimanga essenziale. Per i secondi, invece, la relazione semantica non è orientata e l'inversione dell'ordine non produce necessariamente una differenza di significato (o solo a livello pragmatico-testuale).

Il presente testo è strutturato nel modo seguente: nella sezione 2 presenteremo alcuni sottotipi dei composti trinominali relazionali e analizzeremo la loro struttura interna. Nella sezione 3 presenteremo una tipologia delle teste che determinano la struttura dei composti analizzati basandoci sul materiale tratto dal corpus ItWac. Le conclusioni e le prospettive verranno presentate nella sezione 4.

\section{Struttura e tipologia dei composti $\mathrm{N}-\mathrm{N}-\mathrm{N}$}

\subsection{Diversità dei composti N-N-N}

Il complesso trinominale del tipo relazione governo-sindacati comprende due relazioni di tipo diverso: (a) una relazione di coordinazione tra i nomi-modificatori (governo-sindacati) e (b) una relazione di subordinazione tra il nome-testa da un lato e i suoi modificatori dall'altro (relazione vs. governo-sindacati). La struttura interna può essere concepita in almeno tre modi, come mostrano gli esempi (1a-c).

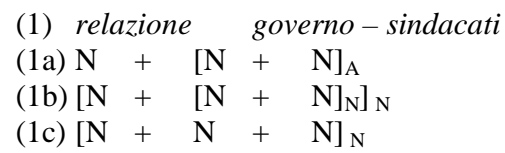

In questa sezione discuteremo in dettaglio le tre possibilità di analisi per vedere quale sia - o quali siano - quelle più appropriate a render conto dei dati. La struttura in (1a) implica che bisognerebbe considerare la relazione di coordinazione $[\mathrm{N}+\mathrm{N}]_{\mathrm{A}}$ (governo-sindacati) come morfologica e la relazione di subordinazione tra relazione e governo-sindacati piuttosto come sintattica, perché sarebbe una relazione di tipo $[\mathrm{N}+\mathrm{A}]$. Le strutture in $(1 \mathrm{~b}-\mathrm{c})$ prevedono invece che ambedue le relazioni siano morfologiche con due ulteriori possibilità: in (1b) si tratterebbe di composizione del tipo $\mathrm{N}+\mathrm{N}$ iterativa, con due strutture $[\mathrm{N}+\mathrm{N}]$ incorporate l'una nell'altra ${ }^{3}$; in (1c) avremmo un solo composto trinominale formato da un'unica regola morfologica.

Alle tre analisi in (1a-c) si fa cenno in L. BAUER (2008:6) e in A. BISETTO (2004:39). A. Bisetto classifica il complesso governo-sindacati come un

\footnotetext{
${ }^{3}$ L'iteratività della struttura è intesa qui in un senso più ampio rispetto alla ricorsività, con riferimento a A. BisETto (2010) piuttosto che a SCALISE e BisetTo (2008:138). In effetti, intendiamo per iteratività un'applicazione doppia dello stesso procedimento di formazione delle parole (p. es. della composizione), come nell'esempio armadio guardaroba di SCALISE e BISETTO (2008 : 138; che gli autori stessi chiamano però ricorsività in quella sede). La ricorsività invece (BISETTO, 2010) sarebbe l'applicazione esatta della stessa regola di composizione, come nel centro trasfusione sangue, dove abbiamo due composti subordinativi incorporati l'uno nell'altro.
} 
composto "N+N coordinativo esocentrico", ma non manca di ricordare che "tali costruzioni [...] non possono mai apparire da sole ma necessitano di un terzo elemento cui appoggiarsi" (p. es. relazione madre-figlio) e aggiunge che dal punto di vista morfosintattico "è particolarmente difficile attribuire categoria lessicale a queste formazioni perché, pur funzionando da modificatori del nome testa, non manifestano proprietà aggettivali." In altri termini, la Bisetto preferisce l'analisi (1b) a quella in (1a). L. BAUER (2008:6) presenta una concezione più diversificata prevedendo una struttura diversa per i composti translative e coparticipant. Per i translative compounds (come il fr. Paris-Rome nel vol ParisRome) propone la struttura (1a), affermando che essi funzionano probabilmente solo come modificatori - e dunque come una specie di "aggettivi composti", . Per quanto riguarda i co-participant compounds (ingl. mother-child relationship), sostiene al contrario che questi ultimi funzionino esclusivamente come modificatori in composti (sic!) più complessi - cioè prevede una concezione come quella in (1b), o eventualmente in (1c).

Comunque stiano le cose, il ragionamento di Bauer implica che i composti trinominali studiati nel presente articolo non devono necessariamente avere tutti la stessa struttura interna. Perciò, prima di procedere a un'analisi strutturale più approfondita, riteniamo utile notare alcune proprietà legate a queste disparità.

Applicando l'ottica di Bauer potremo sostenere che il carattere orientato o meno della relazione tra la coppia di nomi-modificatori coordinati non dipenda dai nomi stessi, bensì dal nome-testa: la relazione semantica nella coppia Parigi-Roma sarà orientata nel viaggio Parigi-Roma e non orientata nel relazioni Parigi-Roma o distanza Parigi-Roma. Infatti, il nome-testa, cioè il N1 nelle lingue romanze ossia il N3 in quelle germaniche, svolge un ruolo particolarmente importante: come ricorda M. MOYNA (2011:50) esso collega sintatticamente i nomi modificatori coordinati, e per alcuni composti del tipo studiato è praticamente obbligatorio dal punto di vista sintattico (cf. anche OLSEN S., 2001 : 299). D'altra parte si può osservare una certa dipendenza tra la natura della testa e il carattere semplice o composto del modificatore: mentre in (2) la testa relazione richiede un complemento composto e rifiuta un complemento nominale semplice, un composto come quello in (3) con la testa capo si comporta nella maniera esattamente opposta.
(2a) relazione governo-sindacati
(2b) relazione governo $*(0)^{5}$
(3a) *apo governo-sindacati
(3b) capo governo

D'ora in poi chiameremo la testa in (2) bivalente e la testa in (3) monovalente, in quanto la prima richiede necessariamente un complemento composto di due

\footnotetext{
${ }^{4}[. .$.$] this type occurs only as a modifying compound, so that it is, in any case, a compound adjective.$ (BAUER L., $2008: 6$ ).

${ }^{5}$ La sequenza relazione governo è grammaticale se relazione viene inteso nel senso di "resoconto scritto".
} 
elementi. Va sottolineato che nei composti di tipo co-participant, i due complementi (governo-sindacati in (2)) non corrispondono in realtà a due argomenti distinti, bensì a un argomento solo, obbligatoriamente composto; le teste in questione possono essere considerate come un sottotipo particolare di predicato semantico a lettura obbligatoriamente collettiva. Nei composti di tipo translative invece, i due complementi (cioè Parigi-Roma in volo Parigi-Roma) corrispondono effettivamente a due argomenti distinti, il che spiega il carattere orientato della loro relazione. ${ }^{6}$ I composti del tipo (3) non corrispondono alle strutture studiate nel presente articolo in quanto non sono trinominali.

Possiamo tuttavia notare che non tutti i composti trinominali sono dotati di una testa bivalente. Per esempio nei composti in (4a, 5a), gli elementi nominali coordinati (autunno-inverno, ambiente-ecologia) possono essere sostituiti da un nome singolo con la stessa funzione $(4 b, 5 b)$.
(4a) collezione autunno-inverno
(5a) commissione ambiente-ecologia
(4b) collezione autunno
(5b) commissione ecologia

Perciò, la testa relazione in (2) è obbligatoriamente bivalente, la testa in (3) è obbligatoriamente monovalente e le teste in (4-5) sono non marcate rispetto a questa proprietà: il loro eventuale carattere bivalente (5a) è facoltativo. Osservazioni simili sono state fatte da S. OLSEN (2004 : 29-30) che descrive i composti del tipo relazione governo-sindacati (identificati qui come composti con testa bivalente) come segue: "embedded compounds [...] function rather as complex arguments in precisely the interpretation required and, hence, also licensed by the meaning of the head. The head can be relational semantically as in the case of 'gap' [...] or can allow a conceptual inference from a collection to its constituent parts [...]. The crucial point is that this type of interpretation is always triggered by a head; such meanings never occur alone". D'altra parte, i composti qui identificati come composti con testa monovalente sono descritti da Olsen (ibid.) nel modo seguente: On the other hand, the meaning that we have isolated for unembedded copulatives - that of a complex concept denoting an ontologically coherent individual - is also possible in embedded environments [...] : "innatistselectivist assumption, speaker-hearer competence, (one-person) writerproducer". Il punto più interessante sollevato da Susan Olsen consiste nella possibilità di una doppia interpretazione dei composti come educator-scientist commission che può essere interpretato sia come un composto a testa bivalente (commission of educators and scientists) che monovalente (commission of educator-scientists).

La distinzione tra l'uso bivalente o monovalente della testa, con la conseguente differenza di interpretazione dei nomi modificatori, può essere messa

\footnotetext{
${ }^{6}$ Il carattere orientato della relazione tra i modificatori potrebbe costituire un argomento per affermare che non si tratta di una relazione coordinativa, almeno dal punto di vista semantico (cf. Haspelmath M., 2004 : 28-32). Preferiamo seguire qui la concezione originale di Bauer, perché la questione sembra piuttosto difficile e spinosa. Infatti, anche con una relazione semanticamente nonorientata possiamo avere occasionalmente strutture sintatticamente orientate (distanza di $A$ da $B$ ) o meno (distanza tra $A$ e $B$ ).
} 
in relazione con l'autonomia potenziale del modificatore. In (4a) con la testa monovalente, il modificatore autunno-inverno è autonomo, nel senso che non deve obbligatoriamente appoggiarsi a un nome-testa. $\mathrm{Si}$ tratta di un composto coordinativo di tipo "additive dvandva" nella classificazione di BAUER (2008), perché il suo significato si riferisce alla somma dei significati delle sue componenti. L'uso autonomo è illustrato dai due esempi seguenti, tratti dal corpus Itwac (6a-b).

(6a) Alla Fortezza da Basso, anche quest'anno, vedremo in anteprima le collezioni di abbigliamento e accessori maschili per l'autunno-inverno 2005 / '06.

(6b) Quando si passa davanti al Bologna 2, soprattutto in autunno-inverno, sembra uno di quei posti tetri e cupi dei romanzi di Stephen King.

Il modificatore ambiente-ecologia in (5a) invece non è un composto autonomo, le sue occorrenze nel corpus Itwac non danno esempi convincenti in cui esso compaia senza appoggiarsi a un nome-testa; lo stesso vale ad esempio per il modificatore del composto legge Bossi-Fini. La non-autonomia di questi composti va di pari passo con la loro natura semantica: essi non corrispondono a composti "additive dvandva" nella classificazione di BAUER (2008), perché non denotano una realtà singola caratterizzata da due proprietà (il signor *Bossi-Fini, a differenza p. es. di Rimskij-Korsakov), ma fanno piuttosto riferimento a due realtà che conservano la loro individualità semantica rispettiva (cf. OLSEN S., $2001: 287,301)$. Si noti che in questi casi, l'interpretazione del fatto che i modificatori ambiente-ecologia o Bossi-Fini costituiscano una coppia è legato alle proprietà della testa. Quest'ultima appare dunque come facoltativamente bivalente (cf. 5a vs. 5b)

La tipologia dei composti finora presentati, rispetto alle proprietà salienti, è riassunta nella tabella 1 .

Tabella 1

\begin{tabular}{|l|l|l|l|l|}
\hline No. & Composto & $\begin{array}{l}\text { Tipo di relazione } \\
\text { coordinativa }\end{array}$ & $\begin{array}{l}\text { Natura della } \\
\text { testa }\end{array}$ & $\begin{array}{l}\text { Natura del } \\
\text { modificatore }\end{array}$ \\
\hline$(7 \mathrm{a})$ & $\begin{array}{l}\text { relazione governo- } \\
\text { sindacati }\end{array}$ & $\begin{array}{l}\text { non-orientata } \\
\text { (co-participant })\end{array}$ & bivalente & non autonomo \\
\hline$(7 \mathrm{~b})$ & viaggio Parigi-Roma & orientata $($ translative $)$ & bivalente & non autonomo \\
\hline$(7 \mathrm{c})$ & $\begin{array}{l}\text { collezione autunno- } \\
\text { inverno }\end{array}$ & non-orientata & monovalente & autonomo \\
\hline$(7 \mathrm{~d})$ & $\begin{array}{l}\text { commissione ambiente- } \\
\text { ecologia }\end{array}$ & non-orientata & mono/bi-valente & non autonomo \\
\hline
\end{tabular}

Nelle sezioni seguenti confronteremo questi casi-tipo con le strutture interne presentate in (1a-c).

\subsection{La struttura $N+[N+N]_{A}$}

Come accennato sopra, l'analisi del tipo $\mathrm{N}+[\mathrm{N}+\mathrm{N}]_{\mathrm{A}}$ considera la relazione di subordinazione tra la testa e i suoi modificatori coordinati come sintattica, prevedendo che l'output del composto N-N coordinativo appartenga alla categoria 
degli aggettivi. Per L. BAUER (2008 : 11-12) potrebbe essere un'analisi adeguata solo per composti di tipo translative (7b).

\begin{tabular}{ll}
\multicolumn{1}{c}{$\mathrm{N}+$} & {$[\mathrm{N}+\mathrm{N}]_{\mathrm{A}}$} \\
(7a) relazione & governo - sindacati \\
(7b) viaggio & Parigi - Roma \\
(7c) collezione & autunno - inverno \\
(7d) commissione & ambiente-ecologia
\end{tabular}

Tale concezione - anche rispetto agli altri casi individuati (7a-7d) - sembra però in italiano contro-intuitiva e poco plausibile per vari motivi. In generale, il complesso $[\mathrm{N}+\mathrm{N}]_{\mathrm{A}}$ infrange la unitary output hypothesis, perché l'esito dei composti N+N in italiano è sempre un nome (SCALISE e MASINI, 2012:78-78). Questo si vede in particolare nel tipo (7c), in cui il modificatore coordinato (autunno-inverno) è un sostantivo che esiste come un nome autonomo (cf. 6a-b).

Per quanto riguarda invece gli altri tipi di composti trinominali $(7 \mathrm{a}, \mathrm{b}, \mathrm{d})$, la non-autonomia del modificatore coordinato è stata rilevata come una prova a favore del suo carattere aggettivale (BAUER, 2008), ma allo stesso tempo è stato sottolineato che il modificatore $[\mathrm{N}+\mathrm{N}]_{\mathrm{A}}$ non presenta altri tratti del comportamento aggettivale (A. BISETTO, $2004: 39$ ). Un problema analogo è stato trattato da D. RICCA (2010:248-250) a proposito dei composti V-N modificatori (tipo /cannonel sparaneve o /nave/ portacontainer). In quel caso, Ricca nota che alcuni composti V-N (come spaccagambe) appaiono esclusivamente come modificatori dei nomi e mai come nomi veri e propri, traendone giustamente la conclusione che il composto V-N in questione si comporta come un aggettivo o, eventualmente, una frase relativa ridotta. Esistono però differenze importanti tra le strutture N-N$\mathrm{N}$ (relazione governo-sindacati) e quelle ( $\mathrm{N}-$ )-V-N (salita spaccagambe). In primo luogo i composti V-N possono esibire altre proprietà tipiche di una distribuzione aggettivale - come p.es. la coordinazione con un aggettivo $(8,9)$, impossibile per le strutture trinominali (10).

(8) Ben diverso, non solo nei fatti ma anche psicologicamente, è andare sotto in modo irregolare, riuscire comunque a pareggiare al $39^{\prime}$ e beccare un altro rigore fasullo $e$ spaccagambe prima del riposo.

(9) Se per i semplici amatori rappresenta un'opportunità di misurarsi su un tracciato decisamente "morbido" e non spaccagambe, per gli agonisti ci sarà la possibilità di confrontarsi con atleti molto quotati.

(10) Le relazioni sociali e *governo-sindacati.

In secondo luogo nei composti V-N modificatori il nome modificato, pur fungendo da testa del sintagma (un cannone sparaneve), viene interpretato come un argomento esterno dell'elemento verbale (spara-); l'interpretazione del composto è quindi dipendente essenzialmente dall'elemento verbale. Nei nostri composti N-N-N invece l'interpretazione dipende esclusivamente dal nome-testa, il che può spiegare sia la sua obbligatorietà sintattica che una certa difficoltà, $o$ addirittura l'impossibilità, di consentire un'interpretazione alle strutture prive del 
nome-testa - indipendentemente dal fatto che la coppia di nomi coordinati venga contestualizzata come un sostantivo (11) o come un suo modificatore (3a).

(11) il/la *governo-sindacati

(3a) *capo governo-sindacati

Per quanto riguarda in particolare i composti di tipo translative (7b), si noti che il comportamento dei modificatori nominali (12a-b) e dei veri e propri modificatori aggettivali (12c-d) è diverso, come mostrano gli esempi seguenti.

(12a) viaggio Parigi-Roma

(12b) viaggio Parigi $-*(0)$

(12c) viaggio ?parigino-romano

(12d) viaggio parigino

Con i modificatori aggettivali (12c-d), la testa viaggio appare come monovalente, mentre risulta bivalente con i modificatori nominali (12a-b).

Per questi motivi il modello $\mathrm{N}+[\mathrm{N}+\mathrm{N}]_{\mathrm{A}}$ ci pare poco adatto alla descrizione dei composti (7a-7d).

\subsection{Le strutture $\left[\mathbf{N}+[\mathbf{N}+\mathbf{N}]_{N}\right]_{N} e[N+N+N]_{N}$}

La struttura del tipo $\left[\mathrm{N}+[\mathrm{N}+\mathrm{N}]_{\mathrm{N}}\right]_{\mathrm{N}}$, suggerita sia da A. Bisetto (2004) che da L. BAUER (2008) per i composti di tipo co-participant, corrisponde sostanzialmente all'analisi adoperata da S. ZUFFI (1981:17-18) per i composti trinominali a modificatore subordinativo (cioè caratterizzato dalla relazione subordinativa tra $\mathrm{N}_{2}-\mathrm{N}_{3}$ ) secondo lo schema seguente:

(13) centro trasfusione sangue

$\left[\begin{array}{lll}\mathrm{N}_{1} & {\left[\mathrm{~N}_{2 \mathrm{a}}+\mathrm{N}_{2 \mathrm{~b}}\right]_{\mathrm{N} 2}}\end{array}\right]$

Il modello in questione è ovviamente adeguato solo per i composti in cui il modificatore $\left[\mathrm{N}_{2 \mathrm{a}}+\mathrm{N}_{2 \mathrm{~b}}\right]_{\mathrm{N} 2}$ è un nome autonomo, indipendente dal nome $\mathrm{N}_{1}$ (centro nel (13)). Dunque, oltre a descrivere bene i citati composti a modificatore subordinativo (trasfusione sangue) sarà adeguato anche per i composti a modificatore coordinativo autonomo. È il caso di collezione autunno-inverno $(7 \mathrm{c})$, in cui la coppia coordinativa (autunno-inverno) è un nome autonomo (14) e occupa un posto riservato normalmente a un nome (15).

(14) Nella foto, un modello di Givenchy per l'autunno-inverno 2003.

(15a) collezione [autunno $]_{\mathrm{N}}$

(15b) collezione [autunno-inverno $]_{\mathrm{N}}$

Tale concezione è conforme anche alle argomentazioni esposte da L. GAETA e D. RICCA (2009:47), secondo cui le strutture N-N subordinative in italiano, ovvero la relazione tra il nome-testa e la coppia dei suoi modificatori coordinati, 
sono risultati di un'operazione morfologica e devono perciò essere considerate come "composti".

Tuttavia, la stessa analisi appare molto meno convincente per i composti in cui la coppia di nomi-modificatori coordinati funziona esclusivamente come modificatore nominale (relazione governo-sindacati, viaggio Parigi-Roma, commissione ambiente-ecologia): siccome il $\mathrm{N}_{2}$ nella struttura $\left[\mathrm{N}_{1}+\left[\mathrm{N}_{2 \mathrm{a}}+\mathrm{N}_{2 \mathrm{~b}}\right]_{\mathrm{N} 2}\right]$ non esiste in isolamento come sostantivo, il nesso $\mathrm{N}_{1}+\mathrm{N}_{2 \mathrm{ab}}$ non può essere descritto come un composto $[\mathrm{N}+\mathrm{N}]_{\mathrm{N}}$. Infatti, i dati ricavati dal corpus Itwac mostrano rari casi in cui il nome-testa può apparire a una certa distanza dai nomimodificatori coordinati $(16,17)$ o figurare in una costruzione sintattica più complessa (18), ma non forniscono esempi convincenti in cui i nomi-modificatori appaino senza il nome-testa (19).

(16) Un altro che ha già conseguenze disastrose e ridimensiona completamente il rapporto tra le parti sociali, governo-sindacati è quello relativo della abolizione del Contratto collettivo nazionale.

(17) il vertice notturno governo-sindacati

(18) E infatti nella Finanziaria, ha ricordato De Mauro, che vanno apportati gli stanziamenti aggiuntivi già previsti per il 2001 e per gli anni successivi, definiti da un tavolo tecnico di governo-sindacati .

(19) il/la *governo-sindacati

Abbiamo visto nella sezione 2.2. che una soluzione possibile è quella di considerare la coppia coordinata $\left[\mathrm{N}_{2 \mathrm{a}}+\mathrm{N}_{2 \mathrm{~b}}\right]$ come un aggettivo $\left(\left[\mathrm{N}_{2 \mathrm{a}}+\mathrm{N}_{2 \mathrm{~b}}\right]_{\mathrm{A}}\right)$, ma le obiezioni non mancano.

Un'analisi alternativa della stessa struttura secondo il modello $[\mathrm{N}+\mathrm{N}+\mathrm{N}]_{\mathrm{N}}$ prevede che tutto il composto trinominale sia un oggetto morfologico solo che si forma in un' unica operazione.

Abbiamo già accennato al fatto che i composti di tipo co-participant (relazione governo-sindacati) e translative (viaggio Parigi-Roma), esemplificati in (7a) e (7b), sono dotati di una testa obbligatoriamente "bivalente", in quanto essa si comporta in un certo senso come un predicato a due posti, perché ammette un modificatore binominale (20a), ma non un modificatore mononominale (21b) con la stessa funzione.

(20a) relazione governo-sindacati

(21b) relazione governo-*(0)

Questo tipo di testa non è eccezionale sia tra i nomi deverbali (alternanza scuola-lavoro, interazione uomo-macchia) che tra nomi astratti (predicativi o relazionali) non-deverbali (conferenza stato-regioni, coppia uomo-donna). L'analisi del tipo $[\mathrm{N}+\mathrm{N}+\mathrm{N}]_{\mathrm{N}}$ rende conto sia della natura bivalente della testa, sia del fatto che la coppia di nomi coordinati governo-sindacati non forma un oggetto morfologico autonomo (22a-b). 
(22a) $\left[[\text { relazione }]_{\mathrm{N} 1 \text {-testa }}[\text { governo }]_{\mathrm{N} 2}-[\text { sindacati }]_{\mathrm{N} 3}\right]_{\mathrm{N}}$

(22b) $\left[[\text { viaggio }]_{\mathrm{N} 1 \text {-testa }}[\text { Parigi }]_{\mathrm{N} 2}-[\text { Roma }]_{\mathrm{N} 3}\right]_{\mathrm{N}}$

$(22 \mathrm{c})\left[[\text { commissione }]_{\mathrm{N} 1 \text {-testa }}[\text { ambiente }]_{\mathrm{N} 2}-[\text { ccologia }]_{\mathrm{N} 3}\right]_{\mathrm{N}}$

Applicheremo la stessa analisi anche ai composti del tipo ((22c, cf. (7d)) con un modificatore non-autonomo e una testa usata come bivalente, nonostante il fatto che il suo carattere bivalente non sia obbligatorio.

\subsection{Conclusione}

Possiamo constatare che i composti $\mathrm{N}_{1}-\mathrm{N}_{2 \mathrm{a}}-\mathrm{N}_{2 b}$ con la relazione coordinativa tra $\mathrm{N}_{2 \mathrm{a}}-\mathrm{N}_{2 \mathrm{~b}}$ e subordinativa tra $\mathrm{N}_{1}-\mathrm{N}_{2}$ mostrano due strutture interne diverse: quella con modificatore incorporato in (23) per i composti in cui il modificatore $\mathrm{N}_{2}$ è autonomo, e quella con il modificatore non-incorporato in (24) per i composti in cui la coppia di nomi coordinati non costituiscono un composto autonomo.

(23) $\left[\mathrm{N}_{1-\text { testa }}-\left[\mathrm{N}_{2 \mathrm{a}}-\mathrm{N}_{2 \mathrm{~b}}\right]_{\mathrm{N} 2}\right]_{\mathrm{N}}$

$\left[[\text { collezione }]_{\mathrm{N} 1 \text {-testa }}\left[[\text { autunno }]_{\mathrm{N} 2 \mathrm{a}}-[\text { inverno }]_{\mathrm{N} 2 \mathrm{~b}}\right]_{\mathrm{N} 2}\right]_{\mathrm{N}}$

(24) $\left[\mathrm{N}_{1 \text {-testa }}-\mathrm{N}_{2}-\mathrm{N}_{3}\right]_{\mathrm{N}}$

$\left[[\text { relazione }]_{\mathrm{N} 1-\text { testa }}[\text { governo }]_{\mathrm{N} 2}-[\text { sindacati }]_{\mathrm{N} 3}\right]_{\mathrm{N}}$

In entrambi i casi il nome-testa fa parte integrante del composto e il suo ruolo appare essenziale nel senso che determina la funzione della coppia dei modificatori nel suo insieme e precisa anche la natura del rapporto che sussiste all'interno della coppia di modificatori coordinati. L'analisi in (23) con modificatore incorporato si ha quando la testa del composto è monovalente (o quando è usata come tale). La lettura in (24) viene invece attivata dalla natura bivalente della testa del composto.

L'analisi dei dati nel capitolo seguente ci permetterà di osservare più da vicino il ruolo che la testa può instaurare tra i modificatori coordinati del composto trinominale.

\section{Tipologia delle teste}

\subsection{Introduzione}

Nella sezione precedente abbiamo studiato la tipologia delle teste in relazione a tre parametri principali: (a) alla natura monovalente o bivalente della testa, (b) alla relazione orientata vs. non-orientata che la testa instaura tra i nomi modificatori coordinati, e (c) al carattere autonomo o meno della coppia di modificatori coordinati (cf. tab. 1) per descrivere la struttura interna dei composti trinominali. Abbiamo anche accennato al fatto che le teste dei composti trinominali possono essere deverbali o meno, di modo che la relazione di subordinazione tra la testa e i modificatori coordinati può essere sia una relazione argomentale che una relazione di tipo grounding (per la terminologia cf. BARONI et al., 2009 : capitolo 3.3). Nella presente sezione ci baseremo sull'analisi di 100 
teste di composti trinominali con un'alta collocabilità nel corpus ItWac per mostrare l'interazione tra i fattori citati. ${ }^{7}$

\subsection{I composti trinominali con teste deverbali}

Nel caso delle teste deverbali si osserva una relazione argomentale tra la testa e i due complementi coordinati. Rispetto agli argomenti del verbo di origine, i dati mostrano la tipologia seguente:

Tabella 2 - Teste bivalenti di composti trinominali (relazione argomentale)

\begin{tabular}{|l|l|l|}
\hline $\begin{array}{l}\text { Ruolo dei complementi N2- } \\
\text { N3 }\end{array}$ & Composto & Struttura soggiacente \\
\hline Oggetto & legame causa-effetto & $\begin{array}{l}\text { la causa e l'effetto sono } \\
\text { legati }\end{array}$ \\
\hline Soggetto & interazione uomo-macchina & $\begin{array}{l}\text { l'uomo e la macchina } \\
\text { interagiscono }\end{array}$ \\
\hline $\begin{array}{l}\text { Soggetto-oggetto in relazione } \\
\text { conversa }\end{array}$ & incontro governo-sindacati & $\begin{array}{l}\text { il governo incontra } i \\
\text { sindacati } \\
\text { i sindacati incontrano il } \\
\text { governo }\end{array}$ \\
\hline $\begin{array}{l}\text { Complementi locativi dei verbi } \\
\text { di movimento }\end{array}$ & spostamenti casa-lavoro & $\begin{array}{l}\text { PRO si sposta da casa al } \\
\text { lavoro }\end{array}$ \\
\hline
\end{tabular}

Altri esempi del primo tipo, in cui i complementi corrispondono a un complemento interno del verbo, sono: abbinamento cibo-vino, collegamento nordsud, accoppiata ansia-negatività, confronto giudice-alteta, contrapposizione destra-sinistra, integrazione scuola-territorio, connessione parola-immagine, alleanza polo-lega, combinazione mouse-tastiera, opposizione maschio-femmina, distinzione uomo-donna, cambio euro-dollaro, accostamento sindacatoterrorismo, coordinamento stato-regioni o separazione stato-chiesa.

I composti del secondo tipo, in cui i complementi corrispondono a un complemento esterno del verbo, sembrano più rari ma non meno interessanti dal punto di vista teorico, perché violano l'ipotesi secondo cui "una non testa non può soddisfare l'argomento esterno della testa” (SCALISE S., 1994 : 146). Altri esempi sono: comunicazione uomo-macchina, contrasto luce-ombra, collaborazione scuola-famiglia, alternanza scuola-lavoro, cooperazione nord-sud o divario ricchi-poveri.

I composti del terzo tipo, dove i complementi corrispondono a un complemento esterno e interno del verbo in una relazione conversa, sono rarissimi. Anche le teste dell'ultimo tipo, in cui i nomi coordinati N2-N3 corrispondono a un complemento di luogo, si riducono a pochi esempi: percorso casa-scuola, tragitto casa-lavoro, direzione nord-sud o, in maniera metaforica, passaggio lira-euro. In

\footnotetext{
${ }^{7}$ Un' analisi alternativa dei rapporti semantici che la testa instaura tra i nomi coordinati si trova in OLSEN (2001, 2004). Olsen distingue rapporti di tipo "between" (lawyer-client relationship, conservative-liberal split) e rapporti di tipo "collection" (mother-daughter duo, water-alcohol mixture). La fonte in questione appare tuttavia troppo poco dettagliata a questo proposito per permetterci di applicare questo metodo al nostro materiale.

${ }^{8}$ Ovvero in forma attiva: «PRO lega la causa e l'effetto ».
} 
questo tipo, la relazione coordinativa tra il N2-N3 è orientata e nella concezione di L. BAUER (2008 : 11-12) corrispondono a dei translative compounds.

I composti trinominali argomentali identificati nel corpus Itwac hanno essenzialmente teste bivalenti con l'eccezione della testa analisi, rilevata in analisi costi-benefici (cf. analisi costi), analisi costo-efficacia, analisi mezzi-fini, ecc. Tuttavia il carattere polivalente di alcune teste può essere soddisfatto da un complemento solo al plurale, come mostra l'esempio (25).

(25a) incontro studente-insegnante

(25b) incontro *insegnante

(25c) incontro insegnanti

Quest'ultimo tipo di testa corrisponde a un normale predicato a lettura obbligatoriamente collettiva (cf. *lo studente s'incontra vs. gli studenti s'incontrano) che richiede un argomento necessariamente plurimo, senza che quest'ultimo debba avere esattamente due componenti (cf. incontro UE-USAONU-Russia sul Mediooriente).

\subsection{I composti trinominali con teste non-deverbali}

Le teste nominali non derivate da verbi esibiscono una relazione di tipo "grounding" con i loro complementi. In alcuni casi, la linea di confine tra le teste deverbali e non-deverbali è piuttosto permeabile, a seconda che si privilegi l'approccio più strettamente diacronico - basato solo sull'etimologia della parola $o$ più sincronico, che dà più peso al legame attuale tra un nome e un verbo nel lessico mentale indipendentemente dall'etimologia del nome. Un caso tipico è rappresentato dai nomi che hanno dato luogo a verbi denominali, come dialogo (> dialogare), confine (> confinare), patto (> pattuire) o miscela (> miscelare). Così ad esempio la testa dialogo, nei composti come dialogo nord-sud o dialogo scuola-famiglia, non ha un'origine deverbale dal punto di vista diacronico, ${ }^{9}$ ma il suo legame con il verbo dialogare si può osservare nel (26a-b).

(26a) dialogo nord-sud

(26b) il nord e il sud dialogano

Sulla stessa scia si possono analizzare nomi che condividono con un verbo lo stesso etimo, come ad esempio parità $(<$ pari) - pareggiare $(<$ pari).

Meno trasparente e, in termini sincronici, poco rilevante sarà l'etimologia deverbale dei nomi il cui etimo verbale è esistito solo in latino e non esiste più in italiano, come connubio. ${ }^{10}$ Discutibili sono pure i casi di nomi deverbali come rapporto (<rapportare) che si sono semanticamente allontanati dalla base

\footnotetext{
${ }^{9}$ L'etimologia indicata nello Zingarelli (2011) è : vc. dotta, lat. diălogu(m), dal gr. diálogos 'discorso (lógos) tra (diá) persone'.

${ }^{10}$ L'etimologia secondo lo Zingarelli (2011) : vc. dotta, lat. conūbiu(m), comp. di cŭm 'con'e nübere 'sposarsi'
} 
verbale, di modo che i complementi dei composti trinominali come rapporto medico-paziente non si possono collegare a un argomento del verbo di origine come nel (26b). Nella presente analisi abbiamo adottato un approccio riduttivo delle teste "deverbali", classificando tutti i casi citati come teste "non-deverbali".

Anche nei composti trinominali di tipo grounding esistono decine di teste bivalenti con cui la coppia N2-N3 costituisce due complementi previsti e deducibili dalle proprietà semantiche della testa N1 (ad esempio: interfaccia uomo-macchina; cf. interfaccia - "ciò che costituisce un collegamento tra due diverse unità”). Pure in questi casi la stessa posizione non può essere normalmente occupata da un nome singolo con lo stesso legame sintatticosemantico con la testa (*interfaccia uomo), ma visto il carattere imprevedibile della relazione grounding, uno dei membri coordinati può essere sottinteso (interfaccia web, interfaccia utenti).

La lista di teste con la più alta collocabilità si trova nella tabella 3.

Tabella 3 - Teste bivalenti di composti trinominali (relazione grounding)

\begin{tabular}{|l|r|r|l|}
\hline \multicolumn{1}{|c|}{ Testa } & $\begin{array}{c}\text { No. di composti } \\
\text { (types) }\end{array}$ & $\begin{array}{c}\text { Frequenza totale } \\
\text { (tokens) }\end{array}$ & Esempio \\
\hline \hline rapporto & 1314 & 4281 & decifit-pil \\
\hline relazione & 279 & 675 & uomo-donna \\
\hline coppia & 134 & 196 & madre-bambino \\
\hline nesso & 91 & 182 & causa-effetto \\
\hline connubio & 78 & 104 & mafia-politica \\
\hline conflitto & 74 & 337 & stato-regioni \\
\hline distanza & 65 & 261 & terra-sole \\
\hline dualismo & 57 & 112 & nord-sud \\
\hline dialogo & 57 & 106 & fede-cultura \\
\hline asse & 56 & 187 & destra-sinistra \\
\hline dicotomia & 55 & 80 & pubblico-privato \\
\hline binomio & 41 & 49 & scienza-tecnica \\
\hline interfaccia & 38 & 104 & uomo-macchina \\
\hline
\end{tabular}

I composti trinominali con la relazione grounding dotati di una testa monovalente mostrano più difficoltà rispetto a un tentativo di descrizione. Il carattere monovalente della testa, derivante dal suo significato, si manifesta nel fatto che la posizione occupata dal composto coordinato può essere occupata anche da un nome solo, con lo stesso legame sintattico-semantico con la testa (27).

(27a) area centro-sud collezione autunno-inverno legge Bossi-Fini 
(27b) area sud

collezione autunno

legge Lupi

Questi composti si suddividono ulteriormente in due grandi categorie. I casi più tipici sono quelli in cui la coppia di modificatori coordinati N2-N3 costituisce un composto coordinativo autonomo, indipendente dalla testa, come in area centro-sud (il centro-sud) o collezione autunno-inverno (l'autunno-inverno). La struttura interna di questi composti è dunque iterativa come nell'esempio (23) ripreso qui sotto - e fa sì che l'intero composto corrisponda piuttosto a una fusione tra due composti binominali anziché a un solo composto trinominale.

\section{(23) $\left[[\text { collezione }]_{\mathrm{N}-\text { testa }}\left[[\text { autunno }]_{\mathrm{N}}-[\text { inverno }]_{\mathrm{N}}\right]_{\mathrm{N}}\right]_{\mathrm{N}}$}

Il secondo tipo, assai più raro, manifesta al contrario una struttura noniterativa, obbligatoriamente trinominale, senza alcuna possibilità di variazione tra gli elementi. Ad esempio il composto modello reddito-spesa, che denota "un modello economico di determinazione del reddito nazionale "11 non comporta un composto autonomo *reddito-spesa, perché quest'ultimo non compare al di fuori del composto trinominale modello reddito-spesa.

Infine si riscontrano vari casi intermedi, tra i quali sono rilevanti i casi di "lessicalizzazione" della testa, come in legge Bossi-Fini - a cui ci si riferisce anche con "la Bossi-Fini" - oppure in rapporto / relazione / dualismo mentecervello che fanno da modello per il composto problema mente-cervello. ${ }^{12}$

Per quanto riguarda il carattere orientato o meno della relazione tra i complementi N2-N3, nei composti con le teste di tipo grounding abbiamo riscontrato solo rarissimi casi di relazione orientata. Sarebbe il caso del nome linea in linea nord-sud. ${ }^{13}$ I composti con la testa non-deverbale corrispondono dunque al tipo co-participant nella classificazione di L. BAUER (2008 : 11-12).

\section{Conclusioni}

L'analisi dei composti $\mathrm{N}_{1}-\mathrm{N}_{2 \mathrm{a}}-\mathrm{N}_{2 \mathrm{~b}}$, caratterizzati da una relazione di coordinazione tra $\mathrm{i}$ nomi-modificatori $\left(\mathrm{N}_{2 \mathrm{a}}-\mathrm{N}_{2 \mathrm{~b}}\right)$ e da una relazione di subordinazione tra la testa $\left(\mathrm{N}_{1}\right)$ e i modificatori $\left(\mathrm{N}_{2}\right)$, ha mostrato che la struttura in questione presenta in italiano vari sottotipi, differenziati a seconda del tipo e della

\footnotetext{
${ }^{11} \mathrm{http}: / /$ www.okpedia.it/modello_reddito_spesa

12 Enciclopedia Treccani: Il problema mente-cervello può essere esposto come segue. Qual'è l'autentica natura dei vari fenomeni mentali che caratterizzano la coscienza negli esseri umani, e in che modo essi sono correlati ai fenomeni fisici che si manifestano nel corpo umano e nel suo sistema nervoso centrale.

http://www.treccani.it/enciclopedia/il-problema-mente-cervello_(Frontiere_della_Vita)/

${ }^{13}$ Una relazione orientata si ha tra la coppia di aggettivi complementi della testa dizionario, ad esempio dizionario italiano-francese.
} 
funzione della testa $\left(\mathrm{N}_{1}\right)$. I casi più comuni sono presentati nella tabella 4 (nell'appendice).

Facendo astrazione dall'ultima categoria (modello reddito-spesa) che appare come poco produttiva, possiamo notare che gli altri casi si suddividono in due grandi gruppi a seconda del carattere bi/polivalente o monovalente della testa:

a) I composti trinominali con la testa bi/polivalente, chiamati relazionali (relational compounds) da B. WëLCHLI (2005 : 7), mostrano una struttura interna non-iterativa (cf. 15) e i loro complementi non appaiono di norma come parole autonome. La natura della relazione coordinativa tra i componenti $\mathrm{N}_{2 \mathrm{a}}-\mathrm{N}_{2 \mathrm{~b}}$ dipende interamente dalle proprietà semantiche della testa ed è probabilmente per questo motivo che essi non hanno una categoria specifica nella classificazione delle costruzioni coordinative di HASPELMATH (2004), né corrispondono alla struttura regolare dei composti coordinativi dell'area SAE (hyponymic coordinating compounds in Arcodia et al., 2010; cf. anche Arcodia, 2010). L. BAUER (2008: 11-12) classifica le coppie $\mathrm{N}_{2 \mathrm{a}}-\mathrm{N}_{2 \mathrm{~b}}$ come co-participant o translative compounds e le descrive - così come abbiamo fatto in questa sede - come modificatori in composti trinominali.

b) Le strutture trinominali con la testa monovalente mostrano al contrario una struttura interna iterativa (cf. 16) e i loro complementi appaiono come parole composte autonome. Non si tratta perciò di veri e propri composti trinominali, bensì di una fusione di due composti binominali.

Un' analisi dettagliata di ambedue i tipi ha dimostrato che rappresentare i nomi coordinati $\mathrm{N}_{2 \mathrm{a}}-\mathrm{N}_{2 b}$ come un modificatore aggettivale è un modello poco plausibile.

Per quanto riguarda il carattere orientato - non-orientato della relazione tra $\mathrm{i}$ nomi coordinati $\mathrm{N}_{2 \mathrm{a}}-\mathrm{N}_{2 \mathrm{~b}}$, che sta alla base della distinzione tra i co-participant e i translative compounds di Bauer, abbiamo notato che la relazione orientata si trova quasi esclusivamente nei casi in cui i complementi corrispondono a complementi locativi di una testa deverbale.

Alcuni problemi cui abbiamo accennato nel presente testo meriterebbero senza alcun dubbio un' analisi più approfondita. Si tratta per esempio della distinzione tra le teste "polivalenti" che richiedono un argomento a interpretazione collettiva senza ulteriori restrizioni (incontro studenti, incontro Ue-Usa, incontro Ue-Usa$\underline{\mathrm{Onu}}$ ) e le teste "bivalenti" che devono essere completate da un argomento collettivo composto da due membri (coppia madre-bambino).

\section{BIBLIOGRAFIA}

ARCODIA Giorgio F. (2010), Coordinating Compounds. Language and linguistics compass, 4, Blackwell, p. 863-873.

ARCODIA Giorgio F.; GRANDI Nicola; WÄLCHLI Bernhard (2010), Coordination in compounding, in: SCALISE, S. / VogEL, I. (eds), Cross-disciplinary Issues in Compounding, Amsterdam, Benjamins, 2010, p. 177-198.

BAroni Marco; Guevara Emiliano; PIRrelli Vito (2009), Sulla tipologia dei composti $\mathrm{N}+\mathrm{N}$ in italiano: principi categoriali ed evidenza distribuzionale a confronto, in: BenatTI Ruben; FerRARI Giacomo; MosCa Monica (eds), 
Linguistica e modelli tecnologici di ricerca (Atti del 40esimo Congresso della Società di Linguistica Italiana), Roma, Bulzoni, p. 73-95.

BAUER Laurie (2008), Dvandva, Word Structure, I/1, 2008, p. 1-20.

BAUER Laurie (2009), Typology of Compounds, in: LIEBER R; ŠTEKAUER P., The Oxford Handbook of Compounding, Oxford, Oxford University Press, p. 343356.

BISETTO Antonietta (2004), Composizione con elementi italiani, in: GROSSMANN, Maria \& RAINER, Franz (a cura di, 2004).

BISETTO Antonietta (2010), Recursiveness and Italian compounds. SKASE Journal of Theoretical Linguistics [online], 2010, vol. 7, $\mathrm{n}^{\circ}$ 1, p. 14-35, disponibile online: http://www.skase.sk/Volumes/JTL15/pdf_doc/02.pdf [cit. 2010-06-02].

BISETTO Antonietta; SCALISE Sergio (2005), The classification of compounds. Lingue e Linguaggio, 4(2). p. 319-332.

GAETA Livio; RICCA Davide (2009), Composita solvantur: Compounds as lexical units or morphological objects? Rivista di Linguistica, 22/1, pp. 35-70.

GROSSMANN Maria; RAINER Franz (a cura di, 2004), La formazione delle parole in italiano, Tübingen, Max Niemeyer Verlag.

HASPELMATH Martin (2004), Coordinating constructions: An overview, in: Haspelmath M. (ed.), Coordinating constructions, Amsterdam, John Benjamins, p. 3-40.

HASPELMATH Martin (2004), Coordinating constructions: An overview, in: Haspelmath M. (ed.), Coordinating constructions, Amsterdam, John Benjamins, p. 3-40.

MASINI Francesca; ScAlise Sergio (2012), Italian compounds, Probus, 1/24, Berlin, Mouton de Gruyter, p. 61-91.

Moyna María I. (2011), Compound words in Spanish, Amsterdam, John Benjamins.

OLSEN Susan (2001), Copulative compounds: a closer look at the interface between syntax and morphology, in: BoOIJ G., MARLE J., Yearbook of Morphology 2000, Kluwer, p. 279-320.

OLSEN Susan (2004), Coordination in morphology and syntax. The case of copulative compounds, in: MEULEN Alice ter; ABRAHAM Werner, The composition of meaning: from lexeme to discourse, Amsterdam, John Benjamins, p. 17-38.

RICCA Davide (2010), Corpus data and theoretical implications: with special reference to Italian V-N compounds, in: SCALISE S.; VOGEL I. (eds), Crossdisciplinary issues in compounding, Amsterdam, John Benjamins, 2010.

SCALISE Sergio (1994), Morfologia, Bologna, Mulino.

SCALISE Sergio; BISETTO Antonietta (2008), La struttura delle parole, Bologna, Mulino.

WÄLCHLI Bernhard (2005), Co-Compounds and natural coordination, Oxford, Oxford University Press.

ZUFFI Stefano (1981), The nominal composition in Italian. Topics in generative morphology, Journal of Italian Linguistics, 1981/2, p. 1-54 


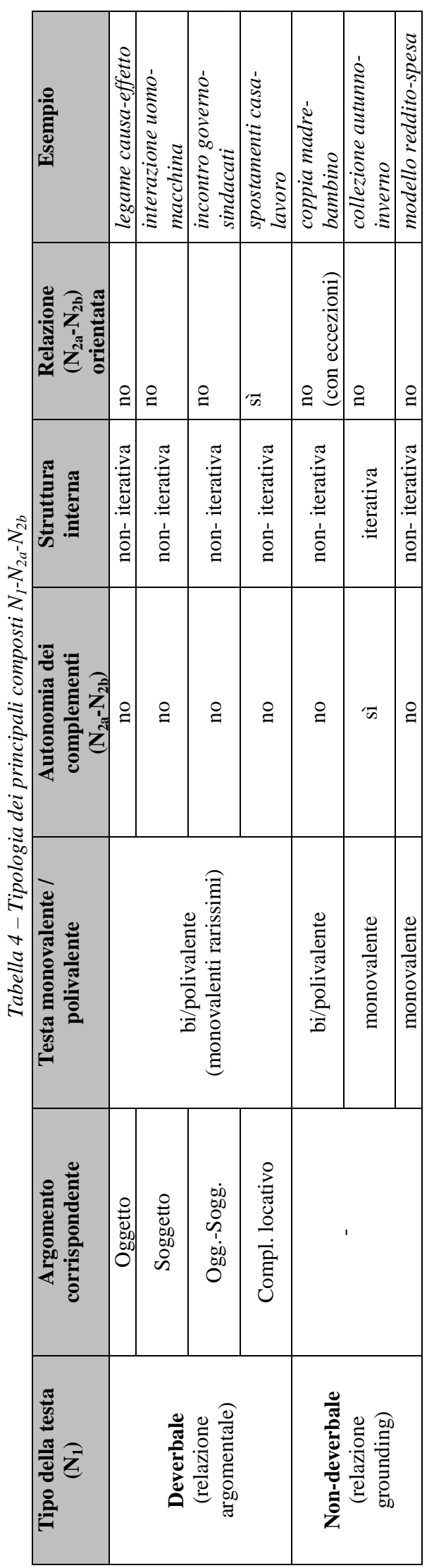

